\title{
Genetic Suppression of Basement Membrane Defects in Caenorhabditis elegans by Gain of Function in Extracellular Matrix and Cell-Matrix Attachment Genes
}

\author{
Jennifer R. Gotenstein, Cassidy C. Koo, Tiffany W. Ho, and Andrew D. Chisholm ${ }^{1}$ \\ Section of Cell and Developmental Biology, Division of Biological Sciences, University of California, San Diego, \\ La Jolla, California 92093
}

ORCID IDs: 0000-0002-7517-1256 (J.R.G.); 0000-0001-5091-0537 (A.D.C.)

\begin{abstract}
Basement membranes are extracellular matrices essential for embryonic development in animals. Peroxidasins are extracellular peroxidases implicated in the unique sulfilimine cross-links between type IV basement membrane collagens. Loss of function in the Caenorhabditis elegans peroxidasin PXN-2 results in fully penetrant embryonic or larval lethality. Using genetic suppressor screening, we find that the requirement for PXN-2 in development can be bypassed by gain of function in multiple genes encoding other basement membrane components, or proteins implicated in cell-matrix attachment. We identify multiple alleles of let-805, encoding the transmembrane protein myotactin, which suppress phenotypes of pxn-2 null mutants and of other basement membrane mutants such as F-spondin/spon-1. These let-805 suppressor alleles cause missense alterations in two pairs of FNIII repeats in the extracellular domain; they act dominantly and have no detectable phenotypes alone, suggesting they cause gain of function. We also identify suppressor missense mutations affecting basement membrane components type IV collagen (emb-9, let-2) and perlecan (unc-52), as well as a mutation affecting spectraplakin (vab-10), a component of the epidermal cytoskeleton. These suppressor alleles do not bypass the developmental requirement for core structural proteins of the basement membrane such as laminin or type IV collagen. In conclusion, putative gain-offunction alterations in matrix proteins or in cell-matrix receptors can overcome the requirement for certain basement membrane proteins in embryonic development, revealing previously unknown plasticity in the genetic requirements for the extracellular matrix.
\end{abstract}

KEYWORDS Peroxidasin; Sulfilimine bond; Myotactin; Spondin; Type IV collagen

ASEMENT membranes (BMs) or basal laminae are specialized extracellular matrices found at the basal surfaces of nearly all animal tissues (Jayadev and Sherwood 2017). BMs are critical for numerous developmental processes, from cell differentiation and specification to organogenesis and tissue growth (Wiradjaja et al. 2010; Sherwood 2015). BMs also function in tissue maintenance and provide a barrier for

Copyright @ 2018 Gotenstein et al

doi: https://doi.org/10.1534/genetics.118.300731

Manuscript received August 30, 2017; accepted for publication February 5, 2018; published Early Online February 12, 2018.

Available freely online through the author-supported open access option.

This is an open-access article distributed under the terms of the Creative Commons Attribution 4.0 International License (http://creativecommons.org/licenses/by/4.0/), which permits unrestricted use, distribution, and reproduction in any medium, provided the original work is properly cited.

Supplemental material is available online at www.genetics.org/lookup/suppl/ doi:10.1534/genetics.118.300731///DC1.

${ }^{1}$ Corresponding author: Division of Biological Sciences, University of California, San Diego, 2402 Bonner Hall, 9500 Gilman Dr., La Jolla, CA 92093-0368. E-mail: adchisholm@ucsd.edu cell invasion as in metastasis of tumor cells. Complete loss of function in BM components often results in embryonic lethality (Smyth et al. 1999), whereas partial loss of function in humans results in a variety of genetic disorders, including muscular dystrophy, epidermolysis bullosa (severe skin blistering), ocular defects, and renal disease, as seen in Alport syndrome (Pozzi et al. 2017).

Assembly of the first BMs in the early embryo involves a series of partly independent yet coordinated self-assembly processes. Laminin, type IV collagen, heparan sulfate proteoglycans (HSPGs) and nidogen are the most prominent and conserved protein components of BMs (Yurchenco 2011; Clay and Sherwood 2015; Fidler et al. 2017). Laminins are required for initial BM assembly, and self-assemble into a cell-associated network independently of type IV collagen, as reviewed in Hohenester and Yurchenco (2013). The laminin network polymerizes in vivo through binding to cell surface receptors, including integrin and dystroglycan; laminin can also be anchored to cells through HSPGs or sulfated glycolipids (Hohenester and Yurchenco 2013). 
Type IV collagen heterotrimers assemble into higher-order networks via lateral associations and covalent cross-links between N-terminal domains (Timpl et al. 1981; Yurchenco and Ruben 1987; Chioran et al. 2017). Additionally, a peroxidasin-mediated sulfilimine bond links the noncollagenous C-terminal (NC1) domains of type IV collagen (Bhave et al. 2012).

In the nematode Caenorhabditis elegans, BMs are present between most tissue types and contain the core components such as laminin and type IV collagens. Loss of laminin causes defects in development beginning in late gastrulation (Huang et al. 2003). C. elegans encodes two type IV collagen $\alpha$-chains, EMB-9 $\alpha 1$ and LET- $2 \alpha 2$, which form $\alpha 1 \alpha 1 \alpha 2$ trimers (Sibley et al. 1994; Gupta et al. 1997; Kubota et al. 2008). Complete loss of type IV collagen function also results in developmental arrest later in embryogenesis; weak hypomorphic alleles of type IV collagen, typically altering a Gly of the Gly-X-Y collagenous motif, result in lower penetrance or temperaturedependent lethality (Gupta et al. 1997).

Studies of human epidermal BM support the hypothesis that type IV collagen networks can form layers separate from laminin networks, and have suggested that these layers are connected by the HSPG perlecan (Behrens et al. 2012). Perlecan has many roles in development and in other processes (Knox and Whitelock 2006). In Drosophila, perlecan localization to BM requires type IV collagen, and perlecan is required to maintain tension of the BM (Pastor-Pareja and Xu 2011). In C. elegans, perlecan does not appear to be a generic BM component (Mullen et al. 1999) but is enriched at surfaces of contractile tissues, and is required for muscle-epidermal attachment (Francis and Waterston 1991; Hresko et al. 1994; Williams and Waterston 1994).

In addition to these core structural components, BMs have additional components that have more regulatory or tissuespecific roles. For example, in C. elegans the specialized BM of the somatic gonad is required for normal gonad development and migration, and contains gonad-specific components such as fibulin, nidogen, and ADAMTS proteases (Kubota et al. $2008,2012)$. In contrast, the specialized BM that transduces force from the body wall muscles to the epidermis contains a distinct subset of proteins, including the F-spondin SPON-1 (Woo et al. 2008). Here we focus on the epidermal-muscle $\mathrm{BM}$ as it relates to embryonic elongation.

Embryonic epidermal morphogenesis and elongation in $C$. elegans involves multiple tissue types (Chisholm and Hardin 2005). Following gastrulation and the enclosure of internal tissues of the embryo by the epidermis in midembryogenesis, the embryo elongates into the tubular shape of larval and adult worms. The epidermis dramatically changes shape during elongation, extending almost threefold in the anteriorposterior axis (Priess and Hirsh 1986). Elongation requires epidermal cytoskeletal proteins (Ding et al. 2004), as well as muscle contraction and an intact epidermal-muscle BM (Williams and Waterston 1994; Chisholm and Hardin 2005).

We previously reported that the $C$. elegans extracellular matrix peroxidase PXN-2/peroxidasin is essential for embryonic development and elongation (Gotenstein et al. 2010).
Peroxidasin was originally identified as a novel matrix peroxidase expressed in Drosophila development (Nelson et al. 1994). Biochemical studies revealed that peroxidasin can catalyze formation of the sulfilimine bonds required for type IV collagen network stabilization (Vanacore et al. 2009; Bhave et al. 2012). In C. elegans, PXN-2/peroxidasin-deficient animals arrest in late embryonic to early larval development, predominantly due to severe muscle-epidermal detachment and aberrant epidermal morphogenesis (Gotenstein et al. 2010). At the muscle-epidermal interface, $p \times n-2$ mutants develop an expanded extracellular matrix, containing multiple electron-dense basement-membrane-like layers. In Drosophila and zebrafish peroxidasin mutants, mechanical forces generated during tissue and organ development cause dissociation of BM and result in failed morphological development (Bhave et al. 2012, 2017; Fidler et al. 2014). Taken together, genetic analysis of peroxidasins is consistent with their proposed biochemical function in type IV collagen cross-linking.

In C. elegans body wall muscles, dense bodies and $\mathrm{M}$ lines are sites of integrin-based muscle adhesions (Francis and Waterston 1991), and are enriched for the BM components UNC-52/perlecan (Rogalski et al. 1993) and EPI-1/laminin $\alpha \mathrm{B}$ (Huang et al. 2003). SPON-1 also localizes to sites of integrin adhesion; spon-1 mutants arrest in late embryonic to early larval stages due to severe muscle detachment and epidermal elongation defects (Woo et al. 2008). SPON-1 does not appear to be required for laminin assembly; instead, SPON-1 may be required for proper integrin-mediated adhesion to the BM. The molecular mechanism of SPON-1 or F-spondin function is not yet understood.

In mammals, $\alpha 6 \beta 4$ integrin and collagen XVII/BP180 cooperatively link the epithelium to BM (Hirako and Owaribe 1998; Zhang and Labouesse 2010). However, the nature of the epidermal cell-matrix attachment in C. elegans has remained unclear. C. elegans encodes a single-pass transmembrane protein, myotactin/LET-805, which localizes to the basal side of the epidermis at structures known as fibrous organelles (Hresko et al. 1999). LET-805 has an extracellular domain (ECD) consisting of numerous fibronectin type III (FNIII) repeats, and is distantly related to FNIII-repeat proteins of the Sidekick family. Null mutations in let-805 result in defective epidermal elongation and fully penetrant embryonic arrest (Hresko et al. 1999). Based on its molecular architecture, localization, and phenotypes, it has been hypothesized that LET-805 might be required for strong attachment of the epidermis to underlying BM. Moreover, both LET-805 and Perlecan display dosage sensitivity in certain genetic backgrounds (Zahreddine et al. 2010). It remains unclear if LET-805 interacts with specific ligands or whether it transduces signals into the epidermis.

Here, we report the results of large-scale screens for suppression of embryonic lethality in pxn-2 or spon-1 mutant backgrounds. We discovered multiple suppressor mutations clustered in a small region of the LET-805 ECD. We also identified missense suppressor mutations in the core BM components type IV collagen and perlecan. Genetic analysis 
is consistent with many of these acting as gain-of-function alleles. These genetic results suggest that compensatory mechanisms exist within the secreted and membrane-bound $\mathrm{BM}$ components that allow the bypass of otherwise essential functions.

\section{Materials and Methods}

\section{Genetics and strain construction}

C. elegans strains were maintained on NGM agar plates at $20-23^{\circ}$ following standard procedures. Bristol N2 was used as wild type for all crosses and scoring. We used the following previously reported mutations and transgenes: pxn-2 (tm3464), pxn-2(ju358, ju432, ju436), pxn-1(ok785), spon1 (ju430ts, e2623), pxn-2+(juEx1044), Ppxn-2-PXN-2(juEx2140) (Gotenstein et al. 2010); vab-10(e698), vab-19(e1036cs) (Ding et al. 2003), emb-9::Dendra (qyIs161) (Ihara et al. 2011); emb9(b189ts), emb-9(g23cg46) (Gupta et al. 1997), let-2(b246) (Sibley et al. 1994) let-2(k193), let-2(k196) (Kubota et al. 2008). Strains used are listed in Supplemental Material, Table S1 in File S1.

vab-10(ju958) arose as a spontaneous suppressor in a pxn2(ju358) strain. let-805 suppressors ju1122 and ju1123 were isolated in a semiclonal screen of $\sim 20,000$ EMS mutagenized haploid genomes for suppression of the null pxn-2(tm3464) mutant to viability in the absence of the pxn-2(+) juEx1044 $\mathrm{GFP}^{+}$rescuing transgenic array. Suppressors ju1165-ju1198 were isolated from a nonclonal screen for EMS-induced suppressors of lethality of the temperature sensitive allele spon-1 (ju430ts). After mutagenesis, $\mathrm{F}_{2}$ and $\mathrm{F}_{3}$ animals were shifted from the permissive $\left(15^{\circ}\right)$ to the nonpermissive $\left(25^{\circ}\right)$ temperature. Surviving animals were propagated to generate suppressed mutant lines. Approximately 40,000 haploid genomes were screened.

\section{CRISPR mutagenesis and GFP knock-in}

Single-guide RNA Cas9 vectors were cloned using the vector Addgene \#47549 and the Quikchange mutagenesis protocol as described (Dickinson et al. 2013), except that Phusion hi-fidelity enzyme and buffer were substituted for Q5; see Table S3 in File S1 for targeting sequences. Point mutations were made using the $d p y-10$ (cn64) co-CRISPR strategy as described (Arribere et al. 2014). A 121 bp oligonucleotide (Ultramer DNA; Integrated DNA Technologies) containing the desired change with 60 bp homology arms was used as repair template for the induced SNPs. Deletions ju1299 and ju1300 were generated in the course of attempting to recreate the vab-10(ju958) SNP. Recreation of the ju1123 T/C (C1424R) SNP to generate ju1386 used the dpy-10(cn64) co-CRISPR strategy with let-805(ju1123) single-guide RNA and oligo repair template.

GFP was inserted at the C-terminus of let-805 in N2 (wild type) and suppressor alleles CZ19652 ju1123, CZ26231 ju1165, and CZ25774 ju1184 using the Self-Excising Drug Selection Cassette as described (Dickinson et al. 2015). Homology arms were cloned into the pDD282 GFP-SEC-3xFlag vector to insert GFP directly before the stop codon. An AvrII/SpeI digest was used to include a flexible linker (RGASGASGAS) before the GFP sequence.

\section{LET-805::GFP image analysis}

For each strain, adult worms grown at $20^{\circ}$ for $24 \mathrm{hr}$ after L4 stage we immobilized on $10 \%$ agarose pads. We took $5 \mu \mathrm{m}$, six-slice z-stack images of the midbody region centered on the vulval/uterine lumen on a Zeiss710 confocal microscope and exported as a maximum intensity projection. LET-805:: GFP aggregation at the uterine seam was quantified using ImageJ particle analysis. A total of 12 images of each genotype were analyzed at $4 \mu \mathrm{m}$ from the GFP localization at the uterine lumen. A region of interest of $30 \times 120$ pixels $(\sim 13 \times$ $52 \mu \mathrm{m}$ ) was used on each side of the uterine seam. A minimum threshold of 35 and a maximum of 255 were used to detect particles. Combined total area of all identified particles in each region of interest is reported.

\section{Transgenes}

The following extrachromosomal transgenes were generated by injections of fosmid or plasmid DNA into N2 worms with Pttx-3-RFP as a co-injection marker: juEx3911-juEx3915 contain let-2 fosmid WRM0640bA06 (10 ng/ $\mu \mathrm{l})$, juEx4356juEx4360 contain emb-9 fosmid WRM0610dH07 (10 ng/ $\mu \mathrm{l})$, and juEx4361-juEx4365 were made by injecting a mix of the above emb-9 and let-2 fosmids (10 $\mathrm{ng} / \mu \mathrm{l}$ each).

\section{Whole genome sequencing and genetic mapping}

Linkage mapping of the pxn-2 suppressor ju958 was performed by crossing pxn-2(tm3464); ju958 suppressed worms to the Hawaiian strain CB4856. ju958 was mapped to chromosome I between N2/CB4856 SNPs located at $~ 11,550 \mathrm{~kb}(+9.17 \mathrm{cM})$ and $12,950 \mathrm{~kb}(+15.72 \mathrm{cM}) \cdot v a b-10$ is at $11,750 \mathrm{~kb}(+9.62$ $\mathrm{cM}$ ). Worm genomic DNA was prepared for sequencing following the Gentra Puregene Tissue Kit DNA purification protocol. Whole genome sequencing (WGS) was performed by BGI, and analyzed as described (McCulloch et al. 2017). Strains were sequenced in batches, using the most outcrossed version of each suppressor available at the time. After the identification of the let-805 "hotspot" regions, all suppressor strains were sequenced specifically in these regions and five new let-805 alleles identified this way (Table 1). Strains in which no mutation was detected in the let-805 hotspots were prepared for WGS.

\section{Phenotypic analysis of suppression}

We quantitated the penetrance of lethal and epidermal morphology defects essentially as described (George et al. 1998). For each genotype, the entire self-progeny brood of $\mathrm{P}_{0}$ animals was quantified $(n=5)$. $P$ values were determined for total lethality using Student's $t$-test, assuming two-tailed unequal variance. To test heterozygous suppression of pxn-2(tm3464), we crossed homozygous suppressed males to transgenically rescued null mutants, pxn-2(tm3464); juEx1044. Broods of 


\begin{tabular}{|c|c|c|c|c|c|c|c|}
\hline Gene & Allele & Chromosome & Residue change & $\begin{array}{c}\text { Codon change } \\
\text { (wild type/mutant) }\end{array}$ & $\begin{array}{c}\text { Mutation } \\
\text { identificationa/strain }\end{array}$ & $\begin{array}{l}\% \text { Lethality } \\
\text { in ju430 }\end{array}$ & $\begin{array}{l}\% \text { Lethality } \\
\text { in tm3464 }\end{array}$ \\
\hline- & - & - & - & - & - & 99.8 & 100 \\
\hline$v a b-10$ & ju958 & I & D941N & Gat/Aat & WGS/mapping CZ13802 & 79.3 & 55.1 \\
\hline$v a b-10$ & ju1299 & I & $\Delta$ D929-A937 & $27 \mathrm{bp} \Delta$ & CRISPR & ND & (31.2 in ju358c) \\
\hline$v a b-10$ & ju1300 & i & $\Delta \mathrm{R} 931-\mathrm{D} 941$ & $33 \mathrm{bp} \Delta$ & CRISPR & ND & (35.1 in ju358c) \\
\hline unc-52 & ju1168 & $\|$ & C402Y & $\mathrm{tGc/tAc}$ & WGS CZ21257 & 6.0 & 2.4 \\
\hline unc-52 & ju1172 & $\|$ & R1442C & $\mathrm{Cgt/Tgt}$ & WGS CZ23560 & 51.0 & 11.4 \\
\hline unc-52 & ju1174 & $\|$ & $\mathrm{R} 175 \mathrm{C}$ & $\mathrm{Cgc/Tgc}$ & WGS CZ21494 & 36.5 & 19.0 \\
\hline unc-52 & ju1188 & $\|$ & E1480K & Gag/Aag & WGS CZ222297 & ND & ND \\
\hline$e m b-9$ & ju1197 & III & P141L & $\mathrm{cCa} / \mathrm{cTa}$ & WGS CZ22017 & 37.2 & 33.8 \\
\hline let-2 & ju1166 & $x$ & E480K & Gaa/Aaa & WGS CZ20580 & 11.0 & 29.2 \\
\hline let-2 & ju1180 & $x$ & P588L & $\mathrm{cCa} / \mathrm{cTa}$ & WGS CZ21496 & 31.2 & 23.1 \\
\hline let-805 & ju1122 & III & E1547K & Gaa/Aaa & WGS CZ18264 & 38.5 & 20.6 \\
\hline let-805 & ju1123 & III & $C 1424 R+R 1584 K$ & $\mathrm{Tgc} / \mathrm{Cgc}+\mathrm{aGa} / \mathrm{aAa}$ & WGS CZ18265 & 25.1 & 6.9 \\
\hline let-805 & ju1386 & III & C1424R & $\mathrm{Tgc} / \mathrm{Cgc}$ & CRISPR & 24.3 & 1.7 \\
\hline let-805 & ju1165 & III & S1594F & $\mathrm{tCc} / \mathrm{tTc}$ & WGS CZ20579 & 8.9 & 4.8 \\
\hline let-805 & ju1167 & III & G1541E & gGa/gAa & WGS CZ20581 & 33.7 & ND \\
\hline let-805 & ju1170 & III & R1076C & $\mathrm{Cgt} / \mathrm{Tgt}$ & WGS CZ20988 & 36.8 & 36.2 \\
\hline let-805 & ju1173 & III & R970C & $\mathrm{Cgt/Tgt}$ & WGS CZ20990 & 9.3 & 61.1 \\
\hline let-805 & ju1175 & III & R1443C & Cgt/Tgt & Resequencing & 44.8 & ND \\
\hline let-805 & ju1177 & III & G1541R & Gga/Aga & Resequencing & 32.1 & ND \\
\hline let-805 & ju1184 & III & G1578E & $\mathrm{gGa} / \mathrm{gAa}$ & Resequencing & 4.5 & 32.6 \\
\hline let-805 & ju1187 & III & P1422R & $\mathrm{cCa} / \mathrm{cGa}$ & WGS CZ22296 & 29.0 & 2.5 \\
\hline let-805 & ju1190 & III & V1052I & Gtc/Atc & Resequencing & 47.1 & ND \\
\hline let-805 & ju1198 & III & A1552V & $\mathrm{gCt} / \mathrm{gTt}$ & Resequencing & 37.4 & ND \\
\hline
\end{tabular}

${ }^{a}$ WGS, Whole genome sequencing. Resequencing, Sanger sequencing of let-805 hotspots.

${ }^{b}$ at $25^{\circ}$.

c ju358 is a viable, strong loss-of-function allele of pxn-2. The single mutant displays $58.5 \%$ lethality.

five nontransgenic $F_{1}$ progeny were quantified at $20^{\circ}$ unless specified. In the case of let-2(ju1166/+), we were unable to isolate viable $F_{1}$ heterozygotes, so broods of nontransgenic $\mathrm{F}_{2} \mathrm{~s}$ from let-2(ju1166/+); pxn-2(tm3464);juEx1044 parent worms were quantified and genotyped to confirm the heterozygosity of parent worms. For complementation testing, single hermaphrodites were mated to homozygous males; four single hermaphrodite crosses were quantified. Quantitative data are in Table S1 in File S1. Suppression of pharynx BM defects was assessed by DIC analysis of day 1 adult worms grown at $20^{\circ}$. Worms were analyzed for bulging in either bulb of the pharynx.

\section{RNA interference}

We performed RNA interference (RNAi) by feeding. Worms were grown at $20^{\circ}$ throughout. L4 worms transferred to $5 \mathrm{mM}$ IPTG, $50 \mu \mathrm{g} / \mathrm{ml}$ carbenicillin plates seeded with RNAi bacteria. After $24 \mathrm{hr}$, three adult worms per genotype and per clone were transferred to individual plates. After $24 \mathrm{hr}$, parent worms were removed from plates. Progeny were counted and phenotypes assessed after an additional $24 \mathrm{hr}$. We used clones from the Ahringer library: clone III-1J01 for let-805 and clone III5A13 for emb-9 (Kamath et al. 2003). A GFP RNAi clone (gift from E. Troemel, University of California, San Diego) was used to target LET-805::GFP. For the lethal pxn-2(tm3464), parent worms expressing a transgenic extrachromosomal array (juEx1044) of cosmid K09C8 (pxn-2) + Psur-5-GFP were plated. Only nontransgenic progeny were assessed.

\section{Protein sequence alignments and structural modeling}

We performed alignments of LET-805 with other FNIII repeat-containing proteins using BLAST. The numbering of FNIII repeats in Figure S2 in File S1 is based on reanalysis with additional protein domain prediction algorithms; sequence alignments use ClustalX. Protein domain graphics were generated using IBS software (Liu et al. 2015), based on domains identified in the conserved domain database of the National Center for Biotechnology Information (Marchler-Bauer et al. 2015). Predicted structures of subsets of the LET-805 FNIII repeats were generated using SWISS-MODEL (Arnold et al. 2006). Models in Figure S3 in File S1 are based on the human IL-6 receptor FNIII repeats.

\section{Data availability}

The authors state that all data necessary for confirming the conclusions presented in the article are represented fully within the article.

\section{Results}

\section{Identification of a novel mutation in spectraplakin vab-10 that suppresses developmental defects of peroxidasin pxn-2 mutants}

We previously reported that $C$. elegans pxn-2 mutants have defective BMs, leading to aberrant tissue morphogenesis and lethality (Gotenstein et al. 2010). pxn-2(ju358) is a partial 
loss of function allele that causes $\sim 60 \%$ embryonic and larval lethality and severe morphology defects (Gotenstein et al. 2010); pxn-2(ju358) homozygotes can be propagated as slow-growing strains. We identified a fast-growing variant strain in the course of routine propagation and confirmed that it retained the original pxn-2(ju358) mutation. This suppressed strain, pxn-2(ju358) sup(ju958), displays $<5 \%$ lethality and almost no detectable morphological defects. Following outcrossing and genetic mapping, we showed that the suppressor ju958 mapped to a $1.4 \mathrm{Mb}$ region on chromosome I. WGS analysis revealed a single missense alteration in this region, affecting $v a b-10$. $v a b-10$ encodes two sets of spectraplakin isoforms, VAB-10A and VAB-10B, each essential for embryonic morphogenesis and epidermal integrity (Bosher et al. 2003). The ju958 missense mutation alters residue D941N in a linker between $\mathrm{N}$-terminal spectrin repeats common to VAB-10A and VAB-10B (Figure 1A). In contrast to vab-10 lossof-function alleles, ju958 single mutants were fully viable and displayed normal morphology.

We found that ju958 was able to suppress the pxn$2(t m 3464)$ null allele [hereafter, $p x n-2(0)]$ to viability (Figure 1B). We previously reported suppression of $p x n-2$ by the superficially wild-type pxn-1 (ok785) null mutation (Gotenstein et al. 2010). Triple mutant vab-10(ju958); pxn1(ok785); pxn-2(0) animals showed additive suppression of pxn-2 (Table S1 in File S1), suggesting that the mechanisms of suppression by pxn-1 and by $v a b-10$ (ju958) are distinct. To test specificity of the suppression for BM defects we tested an additional BM mutant, spon-1 (ju430ts), and found that ju958 partially rescues the conditional lethality of spon-1 (Figure 1B). Thus, suppression by vab-10 (ju958) is not gene specific.

The $v a b$ - 10 locus extends over $45 \mathrm{~kb}$, precluding standard transgenic rescue or reconstruction experiments. To confirm whether the $v a b-10(D 941 N)$ missense alteration was causative for suppression, we performed CRISPR-mediated mutagenesis of wild-type animals at the vab-10(D941) locus and isolated two small, in-frame deletions adjacent to or encompassing the residue affected in ju958 (Figure 1A). These deletions, ju1299 and ju1300, partially suppressed phenotypes of pxn-2(ju358) (Figure 1C), although they did not suppress the pxn-2 null allele to viability. As these deletions also showed suppression of pxn-2(ju358), we conclude that $v a b-10$ mutations in this region are causative for suppression of $p x n-2$ loss of function.

\section{Suppressor screening of pxn-2 and spon-1 identified novel mutations in components of the BM and cell- matrix attachments}

Encouraged by the finding that pxn-2 null mutant phenotypes can be suppressed to viability, we screened directly for EMSinduced suppressors of pxn-2(0) phenotypes (see Materials and Methods) and recovered two suppressors that were identified as alleles of let-805. As described below, these let-805 alleles also suppressed lethal phenotypes of spon-1/F-spondin. We therefore took advantage of the temperature sensitive lethality of a partial loss-of-function allele, spon-1 (ju430ts), to perform a larger-scale selection for suppressors (see Materials and Methods). We recovered 34 additional suppressors of spon-1 (ju430ts). By mapping and WGS, we identified 10 additional alleles of let-805/myotactin, three affecting emb-9 or let-2 (type IV collagen) and four affecting unc-52/perlecan (Table 1). Seventeen additional suppressors will be described elsewhere.

\section{Peroxidasin pxn-2 displays specific genetic interactions with type IV collagen mutations and transgenes}

C. elegans type IV collagen is composed of heterotrimers of two $\alpha$ 1-like (EMB-9) and one $\alpha 2$-like (LET-2) chains (Kramer 2005). Of the three suppressor mutations we identified as affecting type IV collagen chains, two cause missense alterations in LET-2 and one affects EMB-9. In contrast to previously reported loss- or gain-of-function alleles of $C$. elegans type IV collagen (Sibley et al. 1994; Kubota et al. 2008), these suppressor alleles do not cause detectable phenotypes as single mutants, and suppress the lethality of pxn-2 null and of spon-1 loss of function (Figure 2A). All three affect non-Gly residues within the Gly-X-Y repeats of the collagenous $\alpha$-helical domain; two cause Pro-to-Leu missense changes (Figure 2B). In humans, mutations in type IV collagen are known to cause dominantly inherited cerebrovascular disease; a Pro-to-Leu missense change in COL4A1 has been identified in a patient with sporadic cerebral hemorrhage (Weng et al. 2012). This study observed increased intracellular aggregation of this type IV collagen variant, similar to the effects of mutations affecting Gly residues in the Gly-X-Y repeats. In C. elegans, complete loss of function in let-2 or emb-9 causes fully penetrant embryonic lethality, so suppressor alleles of these genes might be either very weak loss of function or gain of function. emb-9(ju1197) fully complemented the lethality of emb-9 null mutants (Table S1 in File S1), consistent with a gain of function. Moreover, emb-9 RNAi abrogated the suppression of pxn-2(0) by emb-9(ju1197) at RNAi dosages that do not cause defects in a wild-type background or enhancement of pxn-2(0) (Figure S1A in File S1). let-2(ju1166)/+ heterozygotes displayed weak suppression (Figure S1B in File S1). Overall, our analysis is most consistent with a gain-of-function model for these suppressor alleles of type IV collagen.

Given the findings that insect and mammalian peroxidasins can promote formation of sulfilimine bonds between type IV collagen NC1 domains in vitro (Bhave et al. 2012), we sought genetic evidence that $C$. elegans pxn-2 functions in type IV collagen cross-linking in vivo. If $\mathrm{PXN}-2$ functions to promote type IV collagen cross-linking, loss of function in pxn-2 and type IV collagen should synergize genetically. The temperaturesensitive allele emb-9(b189) causes $100 \%$ lethality at the restrictive temperature $25^{\circ}$, although it is $98 \%$ viable at $20^{\circ}$. When combined with emb-9(b189), the relatively weak allele pxn-2(ju436) caused a 30-fold increase in lethality at $20^{\circ}$ (Figure 2C). We were unable to test interactions at $25^{\circ}$, as surviving double mutants were sterile. The strongest viable 


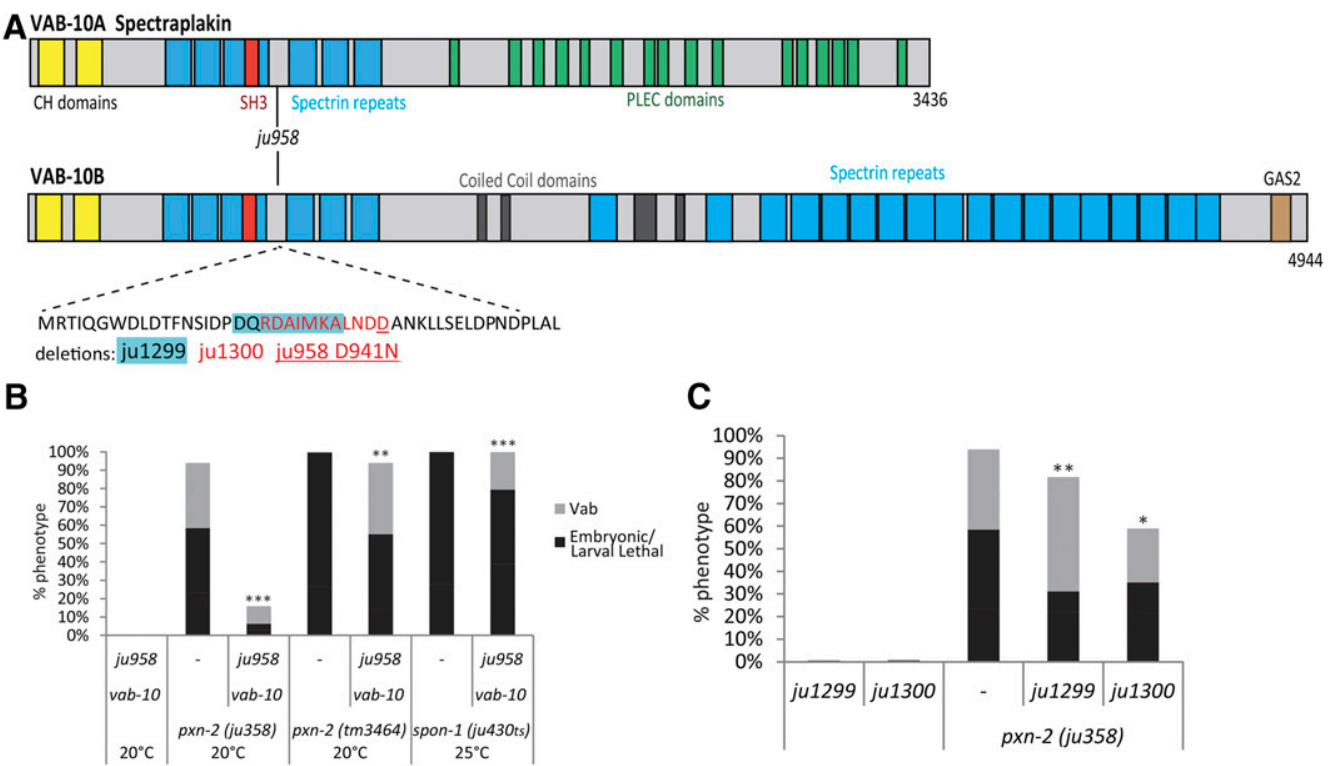

Figure 1 Suppression of BM mutants by a spontaneous mutation in vab-10. (A) Structure of VAB-10 A and $B$ protein domains with ju958 D941N and deletions noted. (B) vab-10(ju958) suppresses pxn-2 null to viability; ju958 partially suppresses spon-1(ju430ts) lethality at nonpermissive temperature. (C) Small in-frame deletions generated by CRISPR near the vab10(ju958) missense residue partly phenocopy the suppression of pxn-2(ju358) by ju958. Results of Student's $t$-test for percent total lethality (sum of embryonic and larval lethality). * $P<0.05$, $* * P<0.01, * * * P<0.001$.

allele pxn-2(ju358) was 100\% lethal in combination with b189: double mutants arrested at the threefold stage of embryogenesis (Figure 2D), similar to pxn-2 (Gotenstein et al. 2010) and emb-9 (Gupta et al. 1997) null mutants. Type IV collagen mutant phenotypes were not modified by loss of function in the other C. elegans peroxidasin gene pxn-1 (Figure 2C).

We observed a similar synergism between a weak allele of $\alpha 2$ type IV collagen, let-2 (b246), with the hypomorphic allele pxn-2(ju436). Double mutant strains were nearly 50\% lethal, whereas single mutants displayed minimal defects. In addition, we tested interaction of a reported type IV collagen gainof-function allele, let-2(k193), which alters an amino acid residue in the NC1 domain, with an intermediate loss-offunction allele pxn-2(ju432) to potentially detect either suppression or synergy. let-2(k193) affects gonad development but does not cause embryonic lethality (Kubota et al. 2008). This type IV collagen allele strongly enhanced pxn-2(ju432) lethality (Table S1 in File S1), similar to the type IV collagen loss-of-function alleles. In contrast, type IV collagen loss-offunction mutations did not display synergistic lethality with a weak allele of spon-1 e2623 (Figure 2C). Collectively, these genetic interactions support the specific synergy of type IV collagen with PXN-2.

We also tested whether overexpression of type IV collagen might bypass the need for $p x n-2$. Among multiple transgenes tested, we found that a transgenic array of emb-9(+) fosmid significantly reduced the lethality and morphological defects of a partial loss-of-function mutant pxn-2(ju358) (Figure 2E), although this array was not able to suppress $p \times n-2$ null mutant lethal phenotypes. Transgenes containing the let-2(+) fosmid alone or a combination of let-2 and emb-9 fosmids were less effective at suppressing pxn-2(ju358) phenotypes (Figure 2E), likely due to the 2 EMB-9:1 LET-2 composition of type IV collagen. In contrast, transgenic arrays expressing type IV collagen were unable to suppress spon-1(ju430ts) phenotypes (Figure 2E), suggesting SPON-1 does not directly affect type
IV collagen function. Together with our double mutant analyses, these genetic interactions are consistent with PXN-2 playing a specific role in type IV collagen function in vivo.

\section{Gain-of-function mutations in a critical region of the LET-805 ECD suppress pxn-2 and spon-1 null mutant phenotypes}

We identified 12 suppressor mutations as causing missense alterations in LET-805/myotactin (Figure 3A). The let-805 gene encodes a predicted single-pass transmembrane protein containing fibronectin type III (FNIII) repeats in its ECD (Hresko et al. 1999). Through sequence alignment and domain analysis (see Materials and Methods), we identified four additional FNIII repeats as well as the 32 previously annotated (see Figure S2 in File S1). Strikingly, all 12 of the identified let-805 suppressor alleles affect two pairs of adjacent FNIII repeats (repeats $9+10$ and $14+15$, as numbered in Figure $3 \mathrm{~A}$ and Figure S2A in File S1) in the middle of the ECD. Although the three alleles located in FNIII repeats $9+10$ were generally weaker suppressors than mutations affecting repeats $14+15$, overall these let-805 mutations displayed strong suppression of lethality of null or loss-of-function alleles of both spon-1 and pxn-2 (Figure 3, B and C and Figure 5). Moreover, ju1123 displayed semidominant suppression of pxn-2(0) (Figure S1C in File S1). As loss of function in let-805 causes fully penetrant recessive embryonic lethality (Hresko et al. 1999), we hypothesize ju1123 and other suppressor alleles of let-805 cause a gain of function. Consistent with this interpretation, let-805 RNAi by feeding, which does not cause lethality in a wild-type background, abrogated suppression in a let-805(ju1123); pxn-2(0) background (Figure S1, D and $\mathrm{E}$ in File S1).

To confirm that the let-805 missense changes were causative for genetic suppression, we used CRISPR to recreate the base transition causing the C1424R alteration, one of two missense changes in the original let-805(ju1123) suppressor 

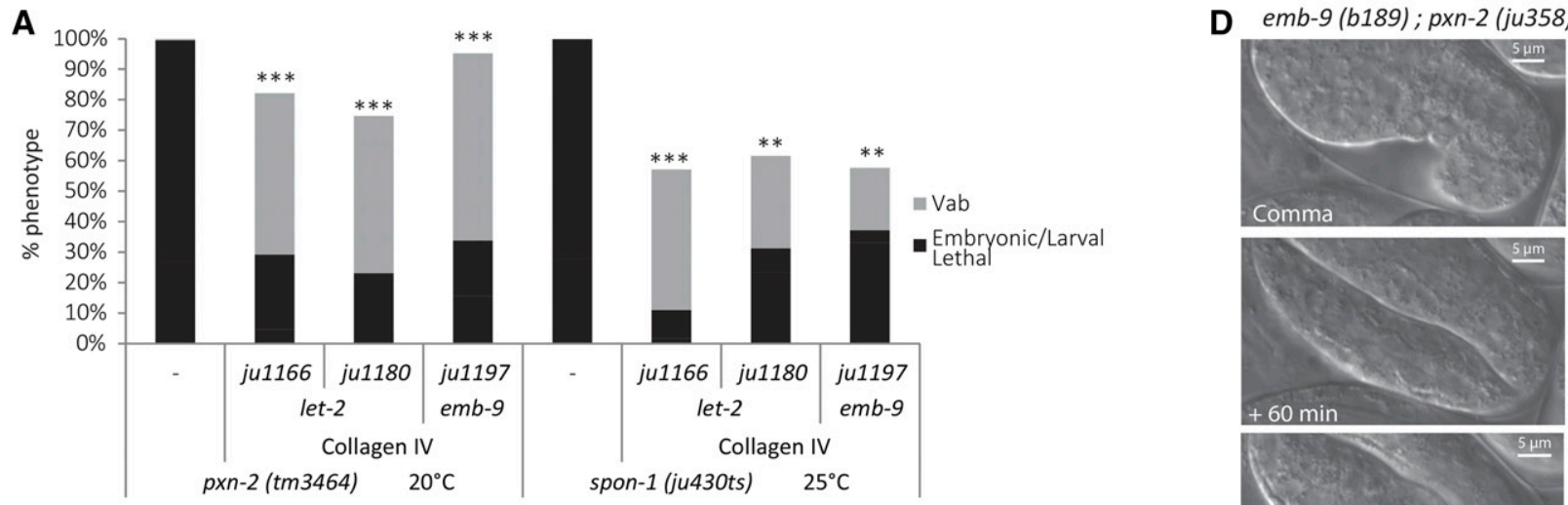

B

emb-9 ju1197 P141L, G213E $\underline{G M P} \underline{G} L \underline{G} P \underline{G} Q \underline{G} \underline{G} Q N \quad \begin{aligned} & \text { G213E } \\ & \text { b189ts }\end{aligned}$
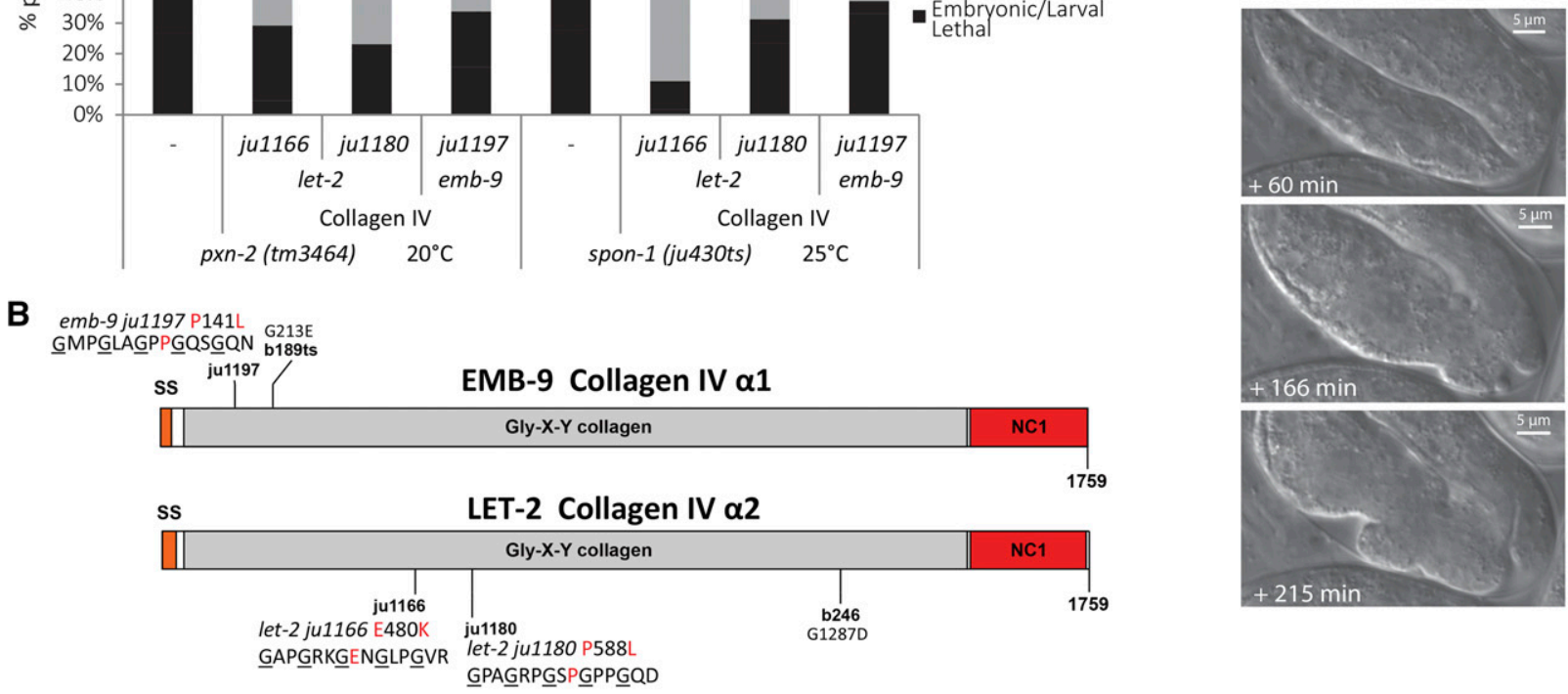

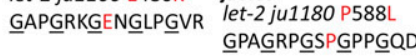
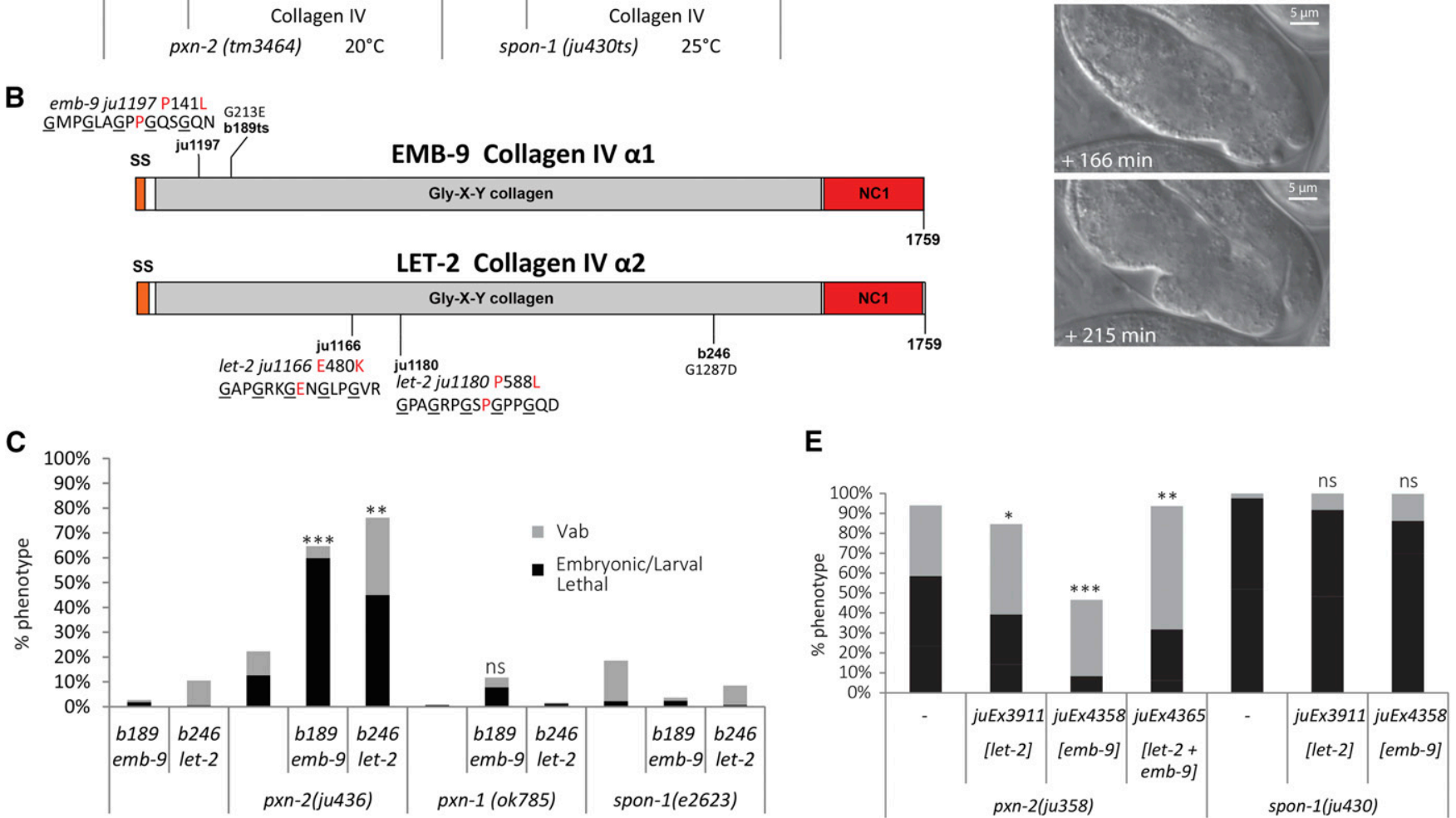

Figure 2 Genetic interactions of type IV collagen mutants with peroxidasin pxn-2. (A) Novel type IV collagen alleles suppress both pxn-2(tm3464) null and spon-1(ju430ts) to viability. (B) Structure of EMB-9 and LET-2 proteins with location of alleles annotated. Novel type IV collagen alleles: ju1166 causes E480K in LET-2, ju1180 causes P588L in LET-2, and ju1197 causes P141L in EMB-9. (C) Loss of function in pxn-2 synergistically enhances the lethality of weak type IV collagen let-2 or emb-9 mutants. A null mutation in pxn-1 does not show synergistic enhancement with type IV collagen alleles. Additionally spon-1 loss of function is unaltered by type IV collagen loss of function. (D) Time series of emb-9(b189); pxn-2(ju358) embryo from comma to arrest. (E) Transgenes containing type IV collagen DNA can partially rescue lethality of pxn-2(If), but not spon-1(If). Results of Student's $t$-test are indicated for percent total lethality (sum of embryonic and larval lethality). $* P<0.05, * * P<0.01, * * * P<0.001$.

strain. The resulting mutant, denoted ju1386, suppressed both spon-1 and pxn-2 phenotypes to levels equivalent to the original let-805(ju1123) suppressor (Figure 3, B and C). These observations confirm that missense alterations in the LET-805 ECD can suppress the requirement for pxn-2 or spon-1 in development.

PXN-2 and SPON-1 likely have distinct molecular roles in the BM; consistent with this, pxn-2 but not spon-1 was suppressed by transgenic overexpression of type IV collagen (Figure 1F). Nevertheless it is striking that almost all the let-805 alleles isolated as suppressors of spon-1(ju430ts) were subsequently found to suppress pxn-2 null phenotypes, suggesting a correlation between suppression of pxn-2 and spon-1. We compared the relative level of suppression of spon-1 and pxn-2, using eight let-805 alleles. Overall there is a correlation between suppression of spon- 1 with suppression of pxn-2 (Figure 3D), with the exception of let-805 (ju1184), which strongly suppresses spon-1 phenotypes but weakly suppresses pxn-2. If ju1184 is excluded, suppression of the two loci is strongly positively correlated, suggestive of a common mechanism of suppression.

To understand how these missense alterations might affect LET-805 structure, we modeled the four FNIII repeats affected by the suppressor mutations (see Materials and Methods) (Figure S3 in File S1). The predicted structures are consistent with these repeats forming a binding interface that 


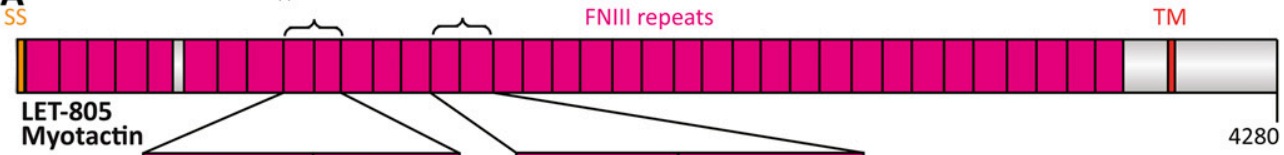

Figure 3 Missense mutations in the let-805 ECD suppress phenotypes due to loss of function in multiple components of the BM. (A) Domain structure of LET-805, indicating the two pairs of FNIII repeats
affected in suppressor alleles. See
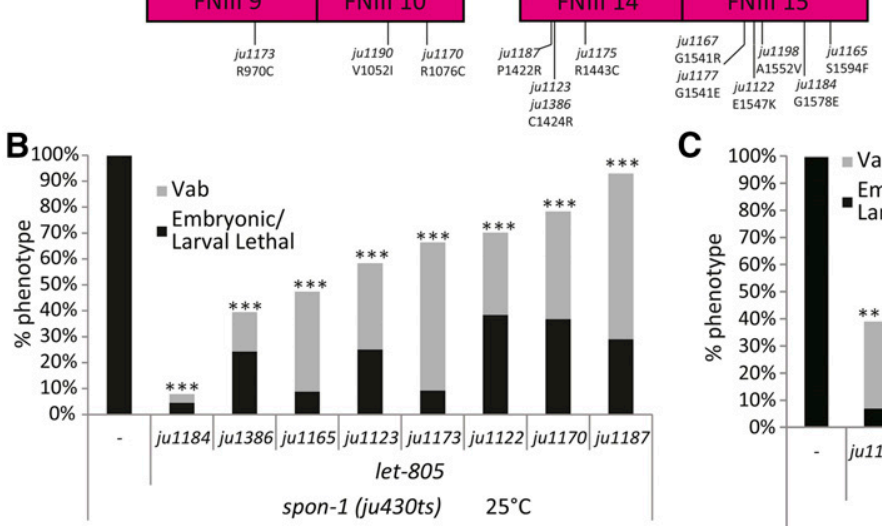

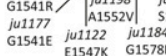

C

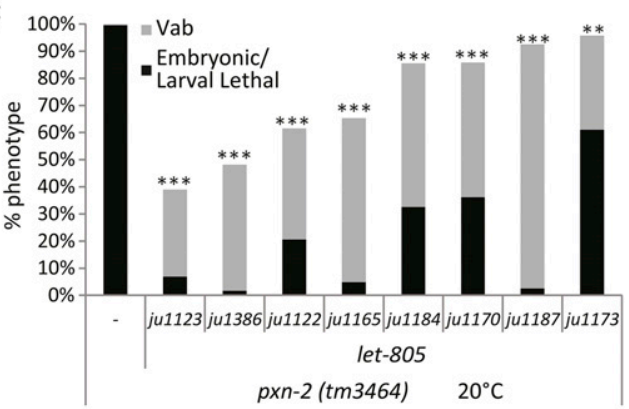
also Figure S2 in File S1. (B) Level of suppression of spon-1(ju430) lethality and $\mathrm{Vab}$ phenotypes by let-805 alleles at $25^{\circ}$. (C) Level of suppression of pxn-2(tm3464) null mutant lethality and Vab phenotypes by let-805 alleles at $20^{\circ}$. (D) Correlation of strength of suppression of spon-1 vs. pxn-2 by let-805 suppressors. Solid line shows correlation trend line excluding ju1184 (slope $=0.86 \pm 0.40)$; dotted line shows correlation trend line including ju1184 (slope $=0.19 \pm 0.33$ ). Results of Student's t-test for percent total lethality (sum of embryonic and larval lethality). $* * P<$ $0.01, * * * P<0.001$.

is affected by the suppressor missense mutations. The missense alterations could directly alter the affinity of LET-805 for an as-yet unidentified ligand. Alternatively, mutations in this region of the ECD could result in a conformational change that alters LET-805 signaling independent of ligands.

Suppression of pxn-2 or spon-1 lethal phenotypes could be for a number of reasons. We therefore examined whether specific pxn-2 phenotypes attributed to BM defects were altered by the let-805 suppressors. Four-dimensional timelapse analysis of let-805(ju1123) pxn-2(0) animals confirmed that suppressed animals showed restored epidermal morphology and body wall muscle attachment (data not shown). In adults, we observed type IV collagen and found distortions in the structure of BM tracks in pxn-2(ju358) compared to wild type (Figure 4, A and B). These distortions were eliminated in suppressed let-805(ju1123) pxn-2(0) animals (Figure $4 \mathrm{C}$ ). Furthermore, the progressive distortions in pharyngeal morphology of pxn-2(ju358) hypomorphs were fully suppressed by let-805(ju1123) (Figure 4, D-F). Taken together, these analyses are consistent with let-805 alleles suppressing multiple $p x n-2$ mutant phenotypes that result from aberrant BM function.

\section{let-805 alleles suppress phenotypes due to partial loss of function in the BM, but not due to loss of function in the epidermal cytoskeleton}

Given the observed synergism of loss-of-function mutations in pxn-2 with type IV collagen, we next tested whether let-805 suppressor alleles could also suppress type IV collagen mutant phenotypes. We found that let-805(ju1123) partially suppressed the lethality of emb-9(b189ts) at the intermediate temperature of $22.5^{\circ}$ (Figure 5), but did not suppress the sterility of b189 mutants shifted to $25^{\circ}$. let-805(ju1123) also suppressed the lethality of let-2(b246ts) at the intermediate temperature of $22.5^{\circ}$, but did not significantly suppress $b 246$ at the restrictive temperature of $25^{\circ}$ (Figure 5). let-805(ju1123) did not suppress either emb-9(tk75gf) or let-2(k193gf) (Figure 5). To address whether let-805 suppressor alleles bypass the requirement for type IV collagen, we attempted to test a null allele emb-9 $(\mathrm{g} 23 \mathrm{cg} 46)$ but were unable to generate a viable let-805(ju1123); emb-9(g23cg46) strain. We further found that let-805(ju1123) was able to suppress the phenotypes of a partial loss-of-function mutation in the laminin $\alpha \mathrm{B}$ chain gene epi-1 (gm121) (Forrester and Garriga 1997) (Figure 5). Taken together, these data indicate that the let-805 gain-offunction mutations can suppress defects in BM integrity due to complete loss of function in nonstructural genes ( $p x n-2$, spon-1) or partial loss of function in structural genes (type IV collagens, laminins), but are unable to bypass the requirement for structural BM components.

Given the suppression of BM mutant defects by vab10(ju958), we also tested whether let-805(ju1123) suppressed mutant defects of epidermal cytoskeletal proteins including $v a b-10$ and other components of trans-epidermal attachments, where LET-805 is known to localize. Partial loss of function in genes encoding cytoskeleton components 

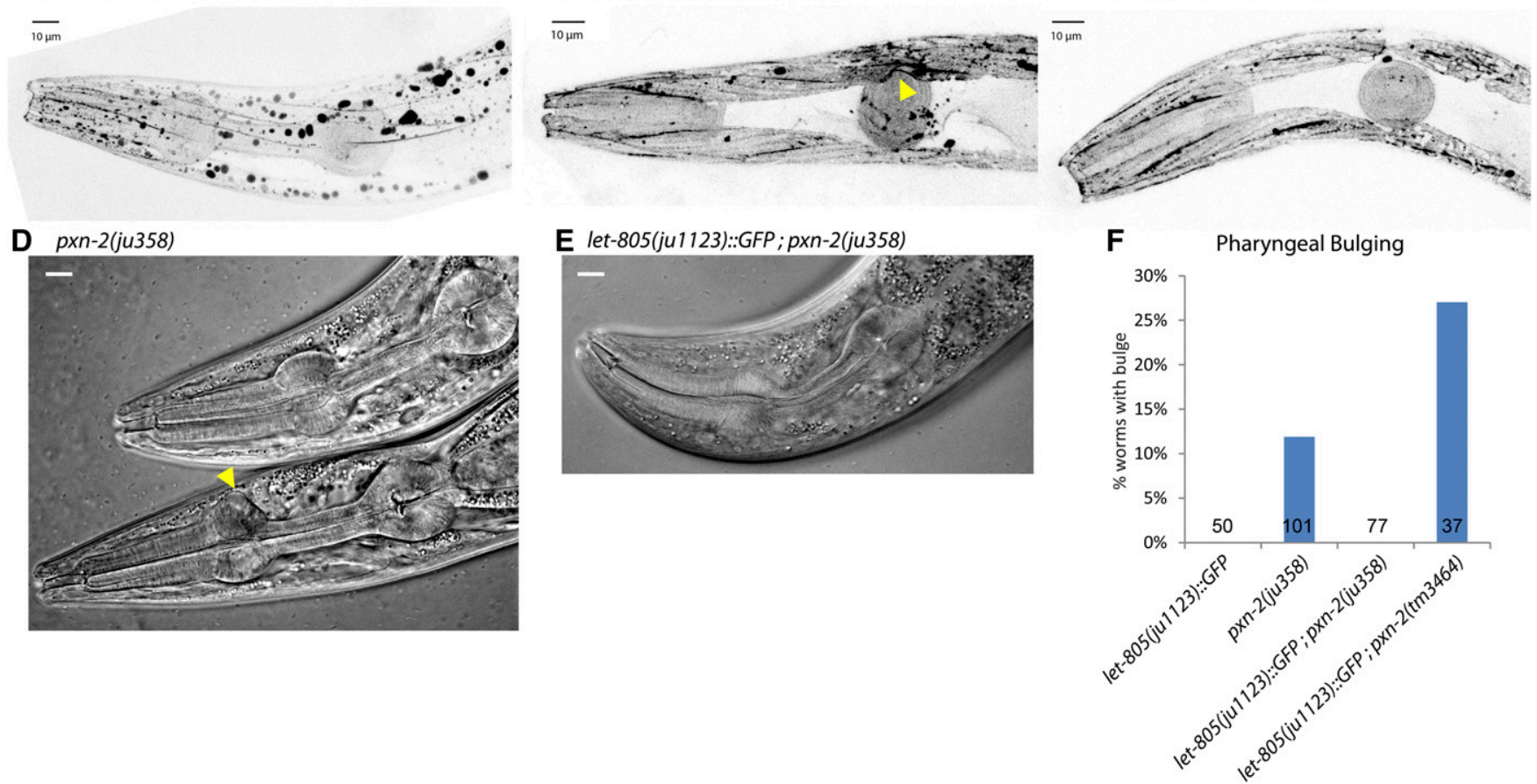

Figure 4 Suppression of BM defects by let-805(ju1123). Functional EMB-9::Dendra (qyls161, fluorescence is shown as inverted, grayscale, projections of z-stacks of superficial sections) localizes to BM overlying body wall muscles and pharynx, as well as accumulating in intracellular aggregates, in wild type (A) and in let-805(ju1123) (data not shown). In pxn-2(ju358), the normal linear localization of EMB-9::Dendra is interrupted (B, arrowhead). (C) EMB-9::Dendra localization is essentially normal in suppressed let-805(ju1123); pxn-2(tm3464) animals. (D-F) Distortions in pharyngeal morphology of pxn-2 young adults (arrowhead, D) are completely suppressed by let-805(ju1123) (image, E and quantitation, F). Scale bars, 10 microns.

(including $v a b-10 /$ spectraplakin, intermediate filament ifb-1, and $v a b-19 /$ Kank) were not suppressed by let-805 suppressor alleles (Figure S4 in File S1), suggesting suppression is specific to defects in BM components.

\section{The LET-805 gain-of-function mutations do not overtly affect protein expression level or localization, but cause aggregation of LET-805::GFP}

To assess whether let-805 missense mutations affect LET-805 localization or levels, we used CRISPR to insert a C-terminal GFP tag in the wild-type, ju1123, ju1165, and ju1184 mutant let-805 loci (see Materials and Methods). All these GFP knock-in animals were fully viable. Wild-type LET-805::GFP localized to epidermal hemidesmosomes, from midembryogenesis onwards (Figure 6), as expected from prior immunocytochemical studies (Francis and Waterston 1991). We also observed localization at the pharynx and uterine seam, consistent with previous results (Francis and Waterston 1991), and along touch neuron processes (Figure 6). These are all regions of the epidermis that require strong adhesive coupling to internal cells. The expression and localization of LET-805(ju1123)::GFP in embryos was overall similar to that of wild-type LET-805::GFP. However, in adults the aggregation of LET-805(ju1123)::GFP at the uterine-epidermal contact was significantly increased compared to the wild type (Figure 6, D-F). Increased aggregation compared to wild type was seen in all three knock-in alleles (Figure 6F). We conclude that the suppressor missense changes do not dramatically affect LET-805 levels or localization, but might enhance its tendency to cluster, consistent with models whereby LET-805 undergoes dimerization or multimerization.

Wild-type LET-805::GFP was severely mislocalized in spon-1 or pxn-2 mutants (Figure S5 in File S1), consistent with the epidermal-muscle detachment observed in these animals (Woo et al. 2008; Gotenstein et al. 2010). The suppressor LET805(ju1123)::GFP strongly restored localization and proper hemidesmosome morphology in pxn-2 or spon-1 mutants, from late embryos through to adults (Figure S5 in File S1), indicating that the GFP tag does not affect the gain of LET-805 function.

\section{Mutations in UNC-52 perlecan can suppress lethality of pxn-2 and spon-1 mutants}

We identified four additional suppressors of spon-1 as affecting the unc-52 gene, encoding the BM component perlecan (Figure 7A). Unlike let-805 suppressor alleles, the unc-52 suppressor alleles affect distinct functional domains across the protein; all four strongly suppress both pxn-2 and spon-1 mutant lethality (Figure 7B). The strongest suppressor unc52(ju1168) affects a laminin-type EGF-like domain. The residue affected in ju1168 is conserved with human HSPG2. In contrast, unc-52(ju1174) affects a nonconserved residue between a conserved Cys and the conserved DXSDE motif in the LDL receptor-like domain of perlecan. Loss of function in specific isoforms of UNC-52 results in adult locomotor defects 


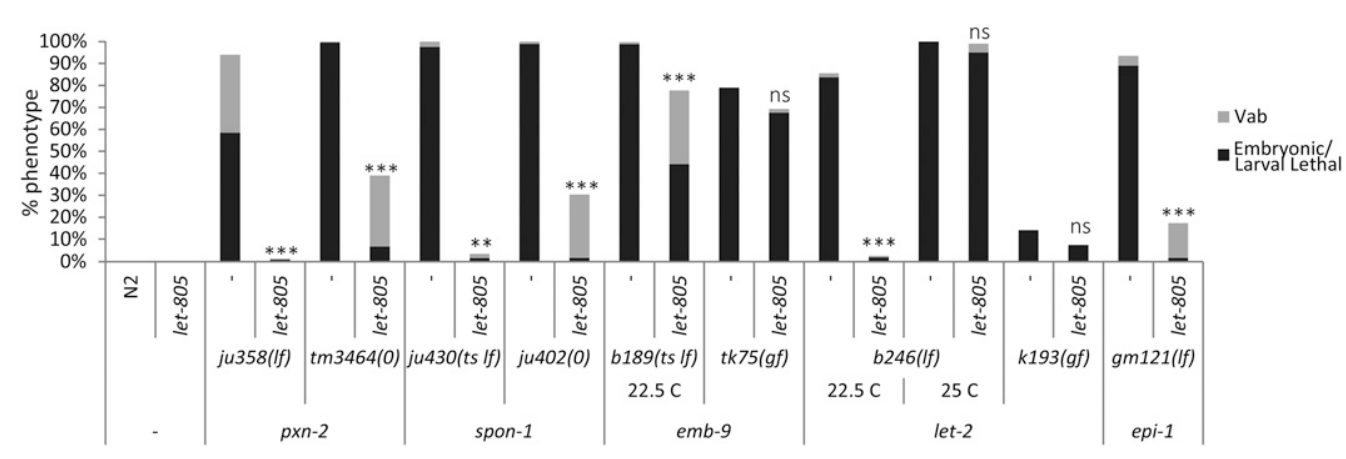

Figure $\mathbf{5}$ let-805(ju1123) suppresses lethality and morphological defects due to partial loss of function in core structural BM components, including the type IV collagen chains emb-9 and let-2 and the laminin subunit epi-1. let805(ju1123) did not suppress defects of emb-9(tk75) or let-2(b246) at the restrictive temperature. Results of Student's $t$-test are indicated for percent total lethality (sum of embryonic and larval lethality). $* * P<0.01, * * * P<0.001$.

(Unc) due to progressive loss of muscle structure (Rogalski et al. 1995). The final two suppressors affect an Ig domain of Perlecan domain IV. Many of the previously identified Unc mutants localize to this region (Mullen et al. 1999). However, unlike previously identified unc-52 variants, the suppressor alleles do not display Unc or Let phenotypes, suggesting unc52 suppressor alleles may cause gain of function or weak loss of function. Consistent with a gain-of-function interpretation, unc-52(ju1168) $/^{+}$heterozygotes displayed semidominant suppression of pxn-2(0) (Figure S1F in File S1). Conversely, the hypomorphic allele unc-52(e444) displayed additive phenotypes when combined with the weak allele pxn-2(ju436) (data not shown), suggesting loss of unc-52 function does not modify pxn-2 phenotypes.

The unc-52 suppressor mutants also partially suppress loss of function in type IV collagen emb-9 (b189) (Figure 7B), comparable to the let-805 suppression of type IV collagen mutants (Figure 5). Previous studies have suggested myotactin/ LET-805 and perlecan/UNC-52 function are closely related (Zahreddine et al. 2010). We therefore tested whether let805 suppressor alleles could suppress unc-52 loss of function. The Unc phenotypes of the partial loss-of-function allele unc52(e444) were unaltered by the let-805(ju1123) suppressor (Table S1 in File S1). We also tested the putative null allele, unc-52(st549) (Rogalski et al. 1995), which causes fully penetrant embryonic lethality, and found that this lethality was not suppressed by let-805(ju1123). This finding suggests that the requirement for certain BM components, such as perlecan, type IV collagen, and laminin, is not bypassed by the kinds of LET-805 altered function mutations described here.

\section{Discussion}

Starting from the chance observation of extragenic suppression of $C$. elegans peroxidasin/pxn-2 phenotypes, we have found that a variety of genes can be mutated to suppress otherwise lethal phenotypes of matrix mutants. Our genetic analysis of $p x n-2$ supports the model that peroxidasins are critical for type IV collagen cross-linking. Nevertheless, the essential role of $p x n-2$ can be bypassed by putative gain-offunction alterations in other essential BM components or in the putative matrix receptor LET-805. These suppressor alleles also suppress phenotypes due to loss of function in SPON-1. As
PXN-2 and SPON-1 likely play different roles in the BM, such suppression may act at the overall level of matrix function rather than compensation for loss of a specific pathway or interaction. These genetic suppression interactions reveal a previously unknown capacity for compensatory interactions between these individually essential genes.

\section{Genetic interactions are consistent with peroxidasin and type IV collagen functioning in a common process}

Our observations of synergism between loss-of-function mutations in the type IV collagen $\alpha 1 \mathrm{emb}-9$ and pxn-2, as well as rescue of $p x n-2$ phenotypes by transgenic overexpression of type IV collagen, is consistent with PXN-2 promoting type IV collagen function in C. elegans. From other biochemical studies, peroxidasins are believed to catalyze sulfilimine cross-links between type IV collagen NC1 domains. In a pxn-2 mutant background, formation of these sulfilimine bonds is presumably reduced, although our efforts to directly assess type IV collagen cross-linking have been unsuccessful, as the embryonic lethality of pxn-2 mutants precludes biochemical analysis. We infer that the combination of partial reduction in type IV collagen cross-linking and partial loss of function in a type IV collagen chain results in severely compromised structural integrity of the BM and subsequent embryonic lethality.

The mechanism by which putative gain-of-function mutations in type IV collagen results in suppression of pxn-2 remains uncertain. The strong suppression of spon- 1 argues against a direct bypass of the pxn-2-mediated sulfilimine bond. Given the location of the type IV collagen suppressor alleles in the collagenous domain, we speculate that the mutations may enhance lateral associations of parallel collagen chains (Yurchenco and Ruben 1987; Knupp and Squire 2005), potentially relaxing the requirement for peroxidasin-mediated cross-linking of the NC1 domains. Hydroxylated proline and/or lysine have been shown to increase the thermostability of collagen (Berg and Prockop 1973). By analogy, a general increase in type IV collagen stability could overcome the need for sulfilimine cross-linking and thus allow genetic suppression of $p x n-2$ phenotypes.

\section{Role of myotactin/LET-805 in BM interactions}

LET-805/myotactin is a giant transmembrane protein whose ECD is composed solely of FNIII repeats. Although myotactin 


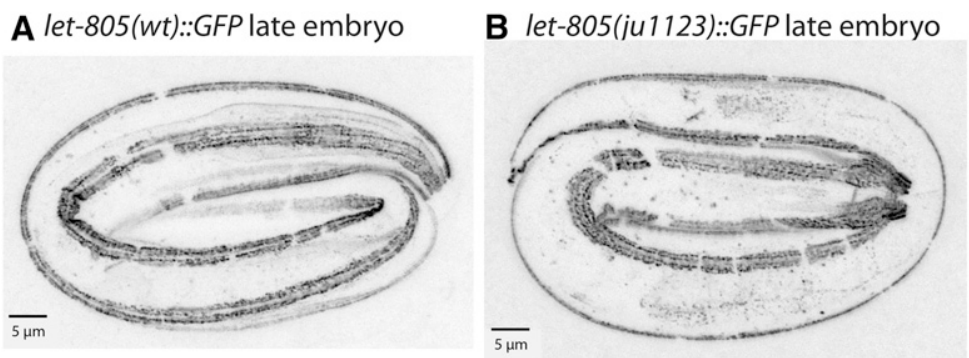

C let-805(wt)::GFP L4 anterior

Figure 6 Localization of LET-805:: GFP knock-ins. In late-stage embryos from (A) wild-type, (B) let805(ju1123), and (C) larval animals, LET-805::GFP localizes to epidermal attachment structures overlying body wall muscles and (D) to the uterine seam. In embryos, LET805::GFP localization in let-805 suppressor mutant backgrounds resembled that of the wild-type LET-805::GFP; in adults mutant for suppressor alleles let-805 (ju1123), let-805(ju1165), and let805(ju1184), LET-805::GFP displays increased aggregates along the mid-body (E) (arrowheads). (F) Quantitation of aggregation as described in Materials and Methods; significance, $t$-test, $* * P<0.01$, $* * * P<0.001$.

E let-805(ju1123)::GFP adult vulva
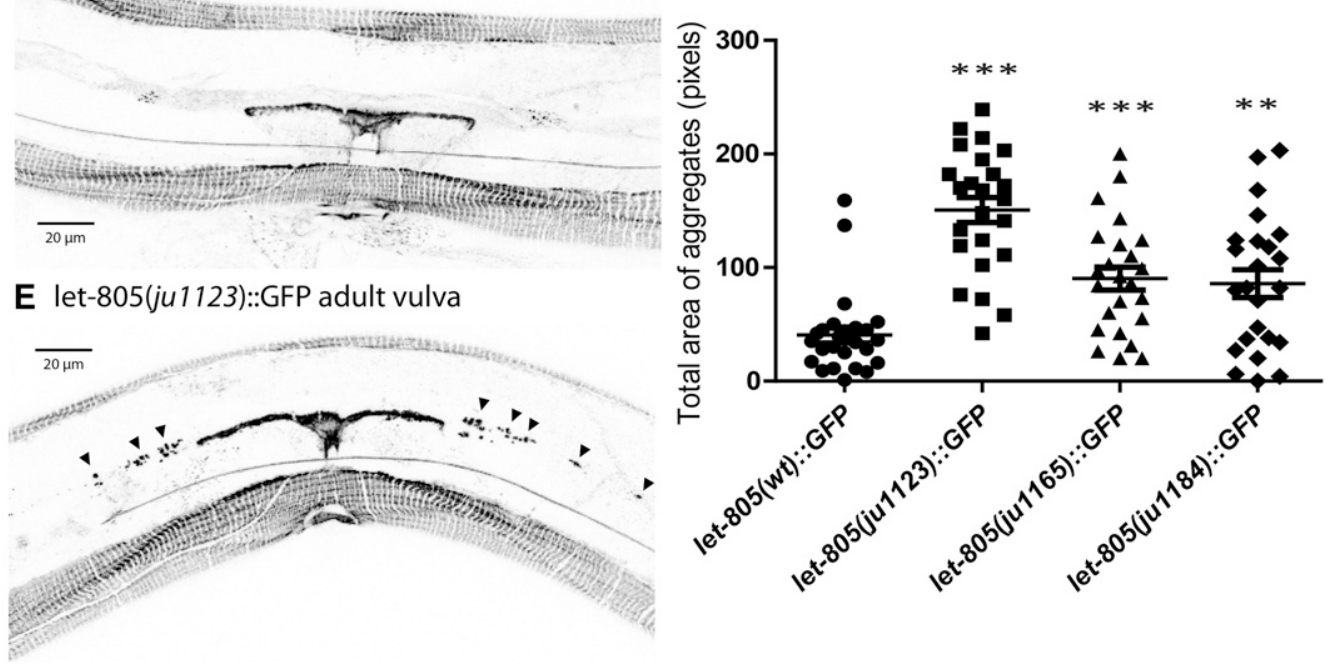

is not conserved outside of nematodes, it has been proposed to play an analogous role to the hemidesmosome transmembrane protein BPAG2 (formerly BP180) (Hresko et al. 1999), which interacts directly with $\alpha 6 \beta 4$ integrin and with the intracellular plakin domains of plectin (Koster et al. 2003). As BPAG2 and myotactin show no sequence similarity, it is unclear whether they share any common mechanisms. It is intriguing that missense alterations in let-805 and the spectraplakin VAB-10 can result in similar genetic suppression of BM defects

The suppressors identified as LET-805 missense changes alter a variety of amino acid residues of its FNIII repeats, in general resulting in either removal of or addition of a charged residue. All residues affected are conserved in the equivalent repeats of myotactin orthologs in other nematodes (Figure S2C in File S1), but are not conserved in the other FNIII repeats of C. elegans myotactin or of other Sidekick family members (Figure S2, A and B in File S1), suggesting these four FNIII repeats may have distinctive roles in myotactin function. The most distinctive features of these repeats are sequences of nonconserved residues between FNIII repeats
8 and 9, and in the middle of FNIII repeat 15 (underlined in red, Figure S2A in File S1). Linker regions between FNIII repeats are important for function in other molecules such as fibronectin itself (Vakonakis et al. 2007). As FNIII repeats are often in large clusters, there are scant examples of single missense alterations having phenotypic consequences. A missense alteration in a FNIII repeat in the PTPRQ transmembrane phosphatase has been linked to deafness (Schraders et al. 2010); how this mutation affects PTPRQ function is not yet known.

Although LET-805 has the structure of a cell-surface receptor protein, it has been unclear whether it interacts with specific ligands, or whether such an interaction might affect intracellular signals. Our identification of the cluster of gainof-function mutations in the ECD as suppressors of BM mutant defects might suggest that this region of LET-805 directly interacts with one or more BM proteins. Our suppression analysis indicates that the let-805 suppressors can suppress phenotypes due to loss of function in multiple BM proteins, but do not suppress null mutations in certain structural BM components. This pattern of suppression argues against a 


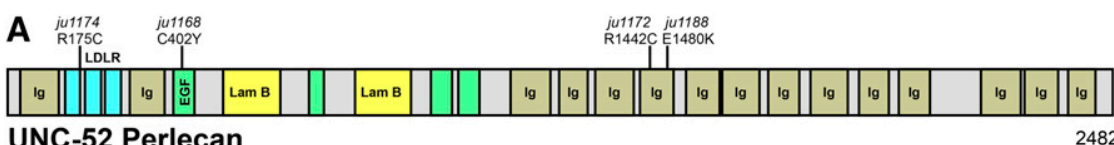

UNC-52 Perlecan

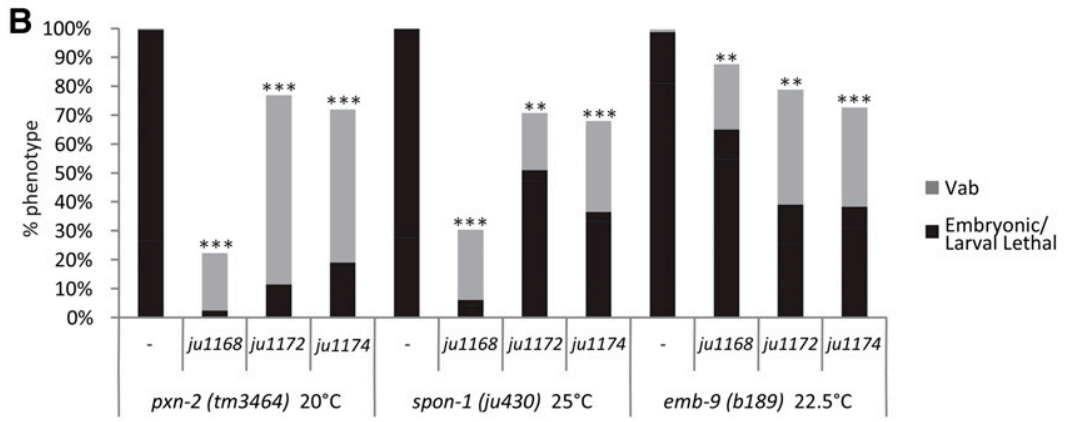

Figure 7 Suppression of BM phenotypes by missense alterations in unc-52 perlecan. (A) Domain structure of UNC-52 (A isoform) and locations of mutations and amino acid substitutions. (B) unc-52 suppressor alleles suppress pxn-2(tm3464) and spon-1(ju430) lethality, and weakly suppress emb-9(b189ts) lethality. LDLR, low-density lipoprotein receptor class A repeat; EGF, Laminin EGF-like domain; Lam B, Laminin B domain. Results of Student's $t$-test for percent total lethality (sum of embryonic and larval lethality). $* * P<0.01$ $* * * P<0.001$ direct interaction mechanism, but is more consistent with bypass suppression in which the functional requirement for certain BM proteins has been relieved.

We consider two general models for how this might occur, although these are not mutually exclusive. In the first model, the gain of function mutations cause suppression by altering or enhancing interactions with specific ligand(s). Although binding partners for the LET-805 ECD are not known, candidates include BM components such as those identified as suppressors in our screen. The clustering of the LET-805 suppressors suggests that they could affect a single binding interaction. Additionally, the aggregation observed in LET805::GFP could indicate an increased binding affinity of the mutant protein for a ligand. The second model is that the gain-of-function mutations promote BM or epidermal function more indirectly, independent of a specific ligand interaction, possibly by stabilizing a specific conformation of LET-805 that enhances its signaling activity. The mechanism of LET-805 activation or regulation is not understood but might involve lateral associations, mediated by its ECD, to form dimeric or higher-order complexes, analogous to other transmembrane receptors. Fibronectin is known interact homophilically, and its FNIII repeats have been hypothesized to be important for forming networks in the extracellular matrix (Ingham et al. 1997). The aggregation of LET-805::GFP might reflect analogous increased selfassociation into higher-order complexes. It remains to be elucidated whether or how LET-805 might signal into cells; it contains a small, highly disordered intracellular domain of unknown function.

\section{Mechanism of suppression by mutations in perlecan}

The third major locus identified in our suppressor screen is unc-52/perlecan, a key conserved molecule in metazoan BMs. Perlecan is a large multidomain HSPG linked to many developmental and physiological processes (Gubbiotti et al. 2017). The five major domains of perlecan all likely function in protein-protein interactions (Iozzo 2005), and the missense mutations identified here could alter affinity of perlecan for specific BM proteins. C. elegans UNC-52 is essential for embryonic development, although it does not appear to be a generic BM component, and it remains unclear if UNC-52 is heparan sulfated. Perlecan incorporation into BMs is thought to be dependent on type IV collagen; genetic analysis has suggested that perlecan can antagonize the function of type IV collagen in the Drosophila BM (Pastor-Pareja and $\mathrm{Xu}$ 2011). Perlecan and type IV collagen also have antagonistic roles in C. elegans synaptic growth (Qin et al. 2014). These observations suggest that the suppressor mutations identified here may not all cause a gain of function, but might weakly reduce function of perlecan.

In summary, we have found that putative gain-of-function alterations in a variety of genes are capable of suppressing profound defects in BM function. These observations suggest that although BM proteins such as SPON-1 and PXN-2 are required for viability in C. elegans, they are not essential for BM formation. Conversely, genetic suppressor analysis suggests that other proteins, such as type IV collagen or perlecan, are essential for BM structure and cannot be bypassed, although partial loss-of-function or hypomorphic alleles of such structural BM components can be bypassed. Although the mechanistic basis of the phenotypic suppression remains to be fully explored, our results suggest unexpected levels of flexibility in the genetic requirements for BM function. It would be interesting to explore if comparable compensatory interactions might occur in the BMs of other species.

\section{Acknowledgments}

We thank members of the A.D. Chisholm and Jin laboratories for advice, discussion, and comments on the manuscript. We thank Kiyoji Nishiwaki (Kwansei Gakuin University) for sharing gain-of-function alleles in emb-9 and let-2, and Sam Cherra for developing the Galaxy workflow for WGS data analysis. Some strains were obtained from the Caenorhabditis Genetics Center, which is funded by the National Institutes of Health (NIH) Office of Research Infrastructure Programs (grant P40 OD010440). This work was supported by NIH grant R01 GM054657 to A.D.C. 


\section{Literature Cited}

Arnold, K., L. Bordoli, J. Kopp, and T. Schwede, 2006 The SWISSMODEL workspace: a web-based environment for protein structure homology modelling. Bioinformatics 22: 195-201. https:// doi.org/10.1093/bioinformatics/bti770

Arribere, J. A., R. T. Bell, B. X. Fu, K. L. Artiles, P. S. Hartman et al., 2014 Efficient marker-free recovery of custom genetic modifications with CRISPR/Cas9 in Caenorhabditis elegans. Genetics 198: 837-846. https://doi.org/10.1534/genetics.114.169730

Behrens, D. T., D. Villone, M. Koch, G. Brunner, L. Sorokin et al., 2012 The epidermal basement membrane is a composite of separate laminin- or collagen IV-containing networks connected by aggregated perlecan, but not by nidogens. J. Biol. Chem. 287: 18700-18709. https://doi.org/10.1074/jbc.M111.336073

Berg, R. A., and D. J. Prockop, 1973 The thermal transition of a non-hydroxylated form of collagen. Evidence for a role for hydroxyproline in stabilizing the triple-helix of collagen. Biochem. Biophys. Res. Commun. 52: 115-120. https://doi.org/10.1016/ 0006-291X(73)90961-3

Bhave, G., C. F. Cummings, R. M. Vanacore, C. Kumagai-Cresse, I. A. Ero-Tolliver et al., 2012 Peroxidasin forms sulfilimine chemical bonds using hypohalous acids in tissue genesis. Nat. Chem. Biol. 8: 784-790. https://doi.org/10.1038/nchembio.1038

Bhave, G., S. Colon, and N. Ferrell, 2017 The sulfilimine crosslink of collagen IV contributes to kidney tubular basement membrane stiffness. Am. J. Physiol. Renal. Physiol. 313: F596-F602. https://doi.org/10.1152/ajprenal.00096.2017

Bosher, J. M., B. S. Hahn, R. Legouis, S. Sookhareea, R. M. Weimer et al., 2003 The Caenorhabditis elegans vab-10 spectraplakin isoforms protect the epidermis against internal and external forces. J. Cell Biol. 161: 757-768. https://doi.org/10.1083/jcb.200302151

Chioran, A., S. Duncan, A. Catalano, T. J. Brown, and M. J. Ringuette, 2017 Collagen IV trafficking: the inside-out and beyond story. Dev. Biol. 431: 124-133. https://doi.org/10.1016/j.ydbio.2017.09.037

Chisholm, A. D., and J. Hardin, 2005 Epidermal morphogenesis (December 01, 2005), WormBook, ed. The C. elegans Research Community, WormBook, doi/10.1895/wormbook.1.35.1, http:// www.wormbook.org.

Clay, M. R., and D. R. Sherwood, 2015 Basement membranes in the worm: a dynamic scaffolding that instructs cellular behaviors and shapes tissues. Curr. Top. Membr. 76: 337-371. https:// doi.org/10.1016/bs.ctm.2015.08.001

Dickinson, D. J., J. D. Ward, D. J. Reiner, and B. Goldstein, 2013 Engineering the Caenorhabditis elegans genome using Cas9-triggered homologous recombination. Nat. Methods 10: 1028-1034. https://doi.org/10.1038/nmeth.2641

Dickinson, D. J., A. M. Pani, J. K. Heppert, C. D. Higgins, and B. Goldstein, 2015 Streamlined genome engineering with a selfexcising drug selection cassette. Genetics 200: 1035-1049. https://doi.org/10.1534/genetics.115.178335

Ding, M., A. Goncharov, Y. Jin, and A. D. Chisholm, 2003 C. elegans ankyrin repeat protein VAB-19 is a component of epidermal attachment structures and is essential for epidermal morphogenesis. Development 130: 5791-5801. https://doi.org/10.1242/ dev.00791

Ding, M., W. M. Woo, and A. D. Chisholm, 2004 The cytoskeleton and epidermal morphogenesis in C. elegans. Exp. Cell Res. 301: 84-90. https://doi.org/10.1016/j.yexcr.2004.08.017

Fidler, A. L., R. M. Vanacore, S. V. Chetyrkin, V. K. Pedchenko, G. Bhave et al., 2014 A unique covalent bond in basement membrane is a primordial innovation for tissue evolution. Proc. Natl. Acad. Sci. USA 111: 331-336. https://doi.org/10.1073/pnas.1318499111

Fidler, A. L., C. E. Darris, S. V. Chetyrkin, V. K. Pedchenko, S. P. Boudko et al., 2017 Collagen IV and basement membrane at the evolutionary dawn of metazoan tissues. eLife 6: e24176.
Forrester, W. C., and G. Garriga, 1997 Genes necessary for C. elegans cell and growth cone migrations. Development 124: 1831-1843.

Francis, R., and R. H. Waterston, 1991 Muscle cell attachment in Caenorhabditis elegans. J. Cell Biol. 114: 465-479. https://doi. org/10.1083/jcb.114.3.465

George, S. E., K. Simokat, J. Hardin, and A. D. Chisholm, 1998 The VAB-1 Eph receptor tyrosine kinase functions in neural and epithelial morphogenesis in C. elegans. Cell 92: 633-643. https:// doi.org/10.1016/S0092-8674(00)81131-9

Gotenstein, J. R., R. E. Swale, T. Fukuda, Z. Wu, C. A. Giurumescu et al., 2010 The C. elegans peroxidasin PXN-2 is essential for embryonic morphogenesis and inhibits adult axon regeneration. Development 137: 3603-3613. https://doi.org/10.1242/ dev.049189

Gubbiotti, M. A., T. Neill, and R. V. Iozzo, 2017 A current view of perlecan in physiology and pathology: a mosaic of functions. Matrix Biol. 57-58: 285-298. https://doi.org/10.1016/j.matbio. 2016.09.003

Gupta, M. C., P. L. Graham, and J. M. Kramer, 1997 Characterization of alpha1(IV) collagen mutations in Caenorhabditis elegans and the effects of alpha1 and alpha2(IV) mutations on type IV collagen distribution. J. Cell Biol. 137: 1185-1196. https://doi.org/ 10.1083/jcb.137.5.1185

Hirako, Y., and K. Owaribe, 1998 Hemidesmosomes and their unique transmembrane protein BP180. Microsc. Res. Tech. 43: 207-217. https://doi.org/10.1002/(SICI)1097-0029(19981101) 43:3<207::AID-JEMT2>3.0.CO;2-Z

Hohenester, E., and P. D. Yurchenco, 2013 Laminins in basement membrane assembly. Cell Adhes. Migr. 7: 56-63. https://doi. org/10.4161/cam.21831

Hresko, M. C., B. D. Williams, and R. H. Waterston, 1994 Assembly of body wall muscle and muscle cell attachment structures in Caenorhabditis elegans. J. Cell Biol. 124: 491-506. https://doi. org/10.1083/jcb.124.4.491

Hresko, M. C., L. A. Schriefer, P. Shrimankar, and R. H. Waterston, 1999 Myotactin, a novel hypodermal protein involved in muscle-cell adhesion in Caenorhabditis elegans. J. Cell Biol. 146: 659-672. https://doi.org/10.1083/jcb.146.3.659

Huang, C. C., D. H. Hall, E. M. Hedgecock, G. Kao, V. Karantza et al., 2003 Laminin alpha subunits and their role in C. elegans development. Development 130: 3343-3358. https://doi.org/ 10.1242/dev.00481

Ihara, S., E. J. Hagedorn, M. A. Morrissey, Q. Chi, F. Motegi et al., 2011 Basement membrane sliding and targeted adhesion remodels tissue boundaries during uterine-vulval attachment in Caenorhabditis elegans. Nat. Cell Biol. 13: 641-651. https:// doi.org/10.1038/ncb2233

Ingham, K. C., S. A. Brew, S. Huff, and S. V. Litvinovich, 1997 Cryptic self-association sites in type III modules of fibronectin. J. Biol. Chem. 272: 1718-1724. https://doi.org/10.1074/jbc.272.3.1718

Iozzo, R. V., 2005 Basement membrane proteoglycans: from cellar to ceiling. Nat. Rev. Mol. Cell Biol. 6: 646-656. https://doi. org/10.1038/nrm1702

Jayadev, R., and D. R. Sherwood, 2017 Basement membranes. Curr. Biol. 20;27(6):R207-R211. https://doi.org/10.1016/j. cub.2017.02.006

Kamath, R. S., A. G. Fraser, Y. Dong, G. Poulin, R. Durbin et al., 2003 Systematic functional analysis of the Caenorhabditis elegans genome using RNAi. Nature 421: 231-237. https://doi. org/10.1038/nature01278

Knox, S. M., and J. M. Whitelock, 2006 Perlecan: how does one molecule do so many things? Cell. Mol. Life Sci. 63: 2435-2445. https://doi.org/10.1007/s00018-006-6162-z

Knupp, C., and J. M. Squire, 2005 Molecular packing in networkforming collagens. Adv. Protein Chem. 70: 375-403. https:// doi.org/10.1016/S0065-3233(05)70011-5 
Koster, J., D. Geerts, B. Favre, L. Borradori, and A. Sonnenberg, 2003 Analysis of the interactions between BP180, BP230, plectin and the integrin alpha6beta4 important for hemidesmosome assembly. J. Cell Sci. 116: 387-399. https://doi.org/10.1242/ jes.00241

Kramer, J. M., 2005 Basement membranes (September 1, 2005), WormBook, ed. The C. elegans Research Community, WormBook, doi/10.1895/wormbook.1.16.1, http:// www.wormbook.org.

Kubota, Y., K. Ohkura, K. K. Tamai, K. Nagata, and K. Nishiwaki, 2008 MIG-17/ADAMTS controls cell migration by recruiting nidogen to the basement membrane in C. elegans. Proc. Natl. Acad. Sci. USA 105: 20804-20809. https://doi.org/10.1073/ pnas.0804055106

Kubota, Y., K. Nagata, A. Sugimoto, and K. Nishiwaki, 2012 Tissue architecture in the Caenorhabditis elegans gonad depends on interactions among fibulin- 1 , type IV collagen and the ADAMTS extracellular protease. Genetics 190: 1379-1388. https://doi. org/10.1534/genetics.111.133173

Liu, W., Y. Xie, J. Ma, X. Luo, P. Nie et al., 2015 IBS: an illustrator for the presentation and visualization of biological sequences. Bioinformatics 31: 3359-3361. https://doi.org/10.1093/bioinformatics/btv362

Marchler-Bauer, A., M. K. Derbyshire, N. R. Gonzales, S. Lu, F. Chitsaz et al., 2015 CDD: NCBI's conserved domain database. Nucleic Acids Res. 43: D222-D226. https://doi.org/10.1093/ nar/gku1221

McCulloch, K. A., Y. B. Qi, S. Takayanagi-Kiya, Y. Jin, and S. J. Cherra, III, 2017 Novel mutations in synaptic transmission genes suppress neuronal hyperexcitation in Caenorhabditis elegans. G3 7: 2055-2063. https://doi.org/10.1534/g3.117.042598

Mullen, G. P., T. M. Rogalski, J. A. Bush, P. R. Gorji, and D. G. Moerman, 1999 Complex patterns of alternative splicing mediate the spatial and temporal distribution of perlecan/UNC-52 in Caenorhabditis elegans. Mol. Biol. Cell 10: 3205-3221. https://doi.org/10.1091/mbc.10.10.3205

Nelson, R. E., L. I. Fessler, Y. Takagi, B. Blumberg, D. R. Keene et al., 1994 Peroxidasin: a novel enzyme-matrix protein of Drosophila development. EMBO J. 13: 3438-3447.

Pastor-Pareja, J. C., and T. Xu, 2011 Shaping cells and organs in Drosophila by opposing roles of fat body-secreted collagen IV and perlecan. Dev. Cell 21: 245-256. https://doi.org/10.1016/ j.devcel.2011.06.026

Pozzi, A., P. D. Yurchenco, and R. V. Iozzo, 2017 The nature and biology of basement membranes. Matrix Biol. 57-58: 1-11. https://doi.org/10.1016/j.matbio.2016.12.009

Priess, J. R., and D. I. Hirsh, 1986 Caenorhabditis elegans morphogenesis: the role of the cytoskeleton in elongation of the embryo. Dev. Biol. 117: 156-173. https://doi.org/10.1016/ 0012-1606(86)90358-1

Qin, J., J. Liang, and M. Ding, 2014 Perlecan antagonizes collagen IV and ADAMTS9/GON-1 in restricting the growth of presynaptic boutons. J. Neurosci. 34: 10311-10324. https://doi.org/ 10.1523/JNEUROSCI.5128-13.2014

Rogalski, T. M., B. D. Williams, G. P. Mullen, and D. G. Moerman, 1993 Products of the unc-52 gene in Caenorhabditis elegans are homologous to the core protein of the mammalian basement membrane heparan sulfate proteoglycan. Genes Dev. 7: 14711484. https://doi.org/10.1101/gad.7.8.1471

Rogalski, T. M., E. J. Gilchrist, G. P. Mullen, and D. G. Moerman, 1995 Mutations in the unc-52 gene responsible for body wall muscle defects in adult Caenorhabditis elegans are located in alternatively spliced exons. Genetics 139: 159-169.

Schraders, M., J. Oostrik, P. L. Huygen, T. M. Strom, E. van Wijk et al., 2010 Mutations in PTPRQ are a cause of autosomalrecessive nonsyndromic hearing impairment DFNB84 and associated with vestibular dysfunction. Am. J. Hum. Genet. 86: 604-610. https://doi.org/10.1016/j.ajhg.2010.02.015

Sherwood, D. R., 2015 A developmental biologist's "outside-thecell" thinking. J. Cell Biol. 210: 369-372. https://doi.org/ 10.1083/jcb.201501083

Sibley, M. H., P. L. Graham, N. von Mende, and J. M. Kramer, 1994 Mutations in the alpha 2(IV) basement membrane collagen gene of Caenorhabditis elegans produce phenotypes of differing severities. EMBO J. 13: 3278-3285.

Smyth, N., H. S. Vatansever, P. Murray, M. Meyer, C. Frie et al., 1999 Absence of basement membranes after targeting the LAMC1 gene results in embryonic lethality due to failure of endoderm differentiation. J. Cell Biol. 144: 151-160. https:// doi.org/10.1083/jcb.144.1.151

Timpl, R., H. Wiedemann, V. Van Delden, H. Furthmayr, and K. Kuhn, 1981 A network model for the organization of type IV collagen molecules in basement membranes. Eur. J. Biochem. 120: 203-211. https://doi.org/10.1111/j.1432-1033.1981. tb05690.x

Vakonakis, I., D. Staunton, L. M. Rooney, and I. D. Campbell, 2007 Interdomain association in fibronectin: insight into cryptic sites and fibrillogenesis. EMBO J. 26: 2575-2583. https:// doi.org/10.1038/sj.emboj.7601694

Vanacore, R., A. J. L. Ham, M. Voehler, C. R. Sanders, T. P. Conrads et al., 2009 A sulfilimine bond identified in collagen IV. Science 325: 1230-1234. https://doi.org/10.1126/science.1176811

Weng, Y. C., A. Sonni, C. Labelle-Dumais, M. de Leau, W. B. Kauffman et al., 2012 COL4A1 mutations in patients with sporadic late-onset intracerebral hemorrhage. Ann. Neurol. 71: 470-477. https://doi.org/10.1002/ana.22682

Williams, B. D., and R. H. Waterston, 1994 Genes critical for muscle development and function in Caenorhabditis elegans identified through lethal mutations. J. Cell Biol. 124: 475490.https://doi.org/10.1083/jcb.124.4.475

Wiradjaja, F., T. DiTommaso, and I. Smyth, 2010 Basement membranes in development and disease. Birth Defects Res. C Embryo Today 90: 8-31. https://doi.org/10.1002/bdrc.20172

Woo, W. M., E. C. Berry, M. L. Hudson, R. E. Swale, A. Goncharov et al., 2008 The C. elegans F-spondin family protein SPON-1 maintains cell adhesion in neural and non-neural tissues. Development 135: 2747-2756. https://doi.org/10.1242/dev.015289

Yurchenco, P. D., 2011 Basement membranes: cell scaffoldings and signaling platforms. Cold Spring Harb. Perspect. Biol. 3: a004911.

Yurchenco, P. D., and G. C. Ruben, 1987 Basement membrane structure in situ: evidence for lateral associations in the type IV collagen network. J. Cell Biol. 105: 2559-2568. https:// doi.org/10.1083/jcb.105.6.2559

Zahreddine, H., H. Zhang, M. Diogon, Y. Nagamatsu, and M. Labouesse, 2010 CRT-1/calreticulin and the E3 ligase EEL-1/ HUWE1 control hemidesmosome maturation in C. elegans development. Curr. Biol. 20: 322-327. https://doi.org/10.1016/ j.cub.2009.12.061

Zhang, H., and M. Labouesse, 2010 The making of hemidesmosome structures in vivo. Dev. Dyn. 239: 1465-1476.

Communicating editor: H. Buelow 